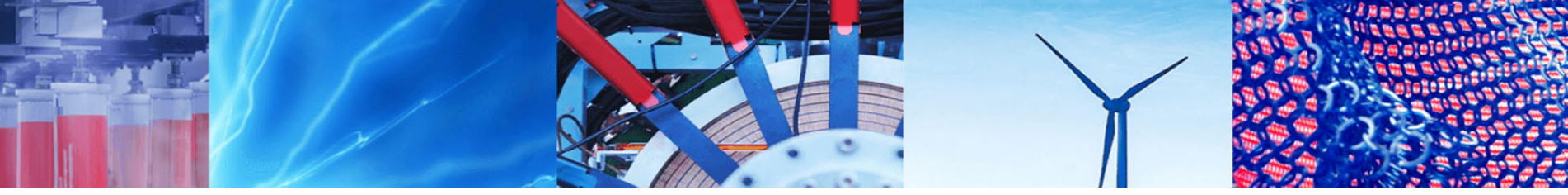

Research Article

\title{
Hunting stability analysis for high-speed trains under crosswinds
}

\author{
Ran-cheng Mao' ${ }^{1}$ Jing Zeng ${ }^{1}$
}

Received: 23 November 2019 / Accepted: 26 March 2020 / Published online: 1 April 2020

(c) Springer Nature Switzerland AG 2020

\begin{abstract}
A 17-DOF mathematical model for a high-speed vehicle under steady crosswinds is established, and the nonlinear wheel/ rail contact geometry and interactive forces are considered. Most importantly, the wheel profiles due to wears with the increase of operating mileage are measured, which have been used to calculate the wheel/rail contact geometry and equivalent conicity. The motion differential equation of the vehicle system is set up, and a program is written to calculate the critical speed under the corresponding parameters considering the vehicle running on straight and curved tracks with crosswind blowing to outer/inner rail. The linearization of the nonlinear vehicle system is conducted, and the critical speed is determined according to the eigenvalues of the linearized system in the equilibrium position. The results demonstrate that the critical speed of the vehicle system is significantly influenced by crosswind. The critical speed increases as the crosswind velocity is increasing when the crosswind blows to inner rail on curves, while it decreases as the crosswind blows to outer rail. And the wears of the wheel profiles also greatly contribute to the hunting stability of the vehicle system due to the resulted high equivalent conicity of wheel/rail contact. The critical speed increases with the increase of curve radius and super-elevation. It is also known through calculations that the derailment safety of the vehicle on curved track is greatly affected by crosswinds.
\end{abstract}

Keywords High-speed vehicle · Crosswinds · Critical speed · Stability · Derailment safety

\section{Introduction}

The stability of railway vehicles is a considerable issue that is decisive to the maximum operating speed for trains running on tracks and it is regarded as an essential index in the assessment of vehicle dynamic behavior. Instability must be prevented in any operation condition, otherwise serious hunting oscillation will occur, which can be extremely detrimental to the operation safety [1]. Meanwhile, regarding the discrepancy of the hunting stability on straight line and curved track, respective studies should be launched with the consideration of their own features. The hunting motion of railway vehicles is closely related to the nonlinear wheel/rail interactive forces, centrifugal forces and crosswind loads on curved tracks, which will determine the equilibrium position of vehicle system during curve negotiation [2]. However, with the continuous increase of train speed, the effects of crosswinds on vehicle stability are gradually obvious, requiring more awareness on the aspects of aerodynamic forces [3].

In the past decades, numerous studies had been carried out by researchers with respect to the hunting stability of railway vehicles. Through linearizing the nonlinear dynamic equations of vehicle system at the equilibrium position, and determining the linear stability by using the eigenvalues of the linearized equations, Kim [4] carried out a parametric study on hunting stability of a full dual-bogie railway vehicle, and analyzed the influence of suspension parameters on linear critical speed. Cheng [5] used the linearization method to analyze the stability of high-speed railway vehicle running on straight line with a half-car model. In addition, Zboinski and Dusza [6]

Jing Zeng, zeng@swjtu.edu.cn; Ran-cheng Mao, mrc.swjtu.edu.cn@my.swjtu.edu.cn | 'State Key Laboratory of Traction Power, Southwest Jiaotong University, Cheng Du, Si Chuan, People's Republic of China. 
implemented the studies of vehicle nonlinear stability on a curved track, and investigated the effects of suspension parameters on the system bifurcation diagram. Followed by the researches considering the effects of crosswinds. Deng et al. [7] compared the difference while the highspeed train running via crosswinds and corresponding traffic safety of both windproof facilities, with aerodynamic loads generated from CFD simulations and safety criterions derived from wind-train-track dynamic analysis system. Cheng et al. [8] established a 24-DOF vehicle system model, and studied the governing differential equations of motion for a tilting railway vehicle moving over irregular curved tracks under wind loads. Baker et al. [9] simulated the dynamic response of vehicle system by considering the crosswind forces. Andreas [10] discussed the feasibility of different approaches to ensure crosswind stability by means of active control, then proposed four different concepts and chose the most promising one based on the data from wind tunnel measurements. Myung et al. [11] predicted the derailment pattern and the critical crosswind speed of the running trains with the consideration of dynamic wheel-rail effects. Choi [12] studied the critical speed of high-speed trains considering wheel-rail contact, indicating that the critical speed increases as the creep curve becomes stiff before saturation, which is more effective than the variation in suspension parameters and the consideration of flange contact can also increase the critical speed. Yan et al. [13] utilized spectral analysis approach to evaluate the overturning risk of high-speed trains under strong crosswinds, demonstrating that the dynamic wheel-rail contact force induced by track irregularities is lower than that by wind turbulence. Xiaohui et al. [14] performed research on hunting stability of vehicles under steady aerodynamic loads, and reached the conclusion that the linear critical speed decreases monotonously as the crosswind velocity increases, and the influences of pitch moment and lift force on the linear critical speed are larger than the other components of the aerodynamic loads. Mao et al. [15] carried out the studies of the influence of crosswind characteristics on aerodynamic forces acted on high-speed train by theoretical analysis, model experiment, air fluid simulation and multi-body system dynamics simulation. Kikuchi et al. [16] obtained the aerodynamic force coefficients from wind tunnel experiment and a full-scale model field test firstly, and then compared with the respective experiments, they also evaluated the effects of aerodynamic force coefficients by using the critical wind speed determined from the aerodynamic force coefficients. Zhuang and Lu [17] studied the yaw effect of the side flow around a high-speed train by means of large eddy simulation, and concluded that the time-dependent aerodynamic forces are dominated by several energetic frequencies and the frequency range is broadened to a higher extent for the large yaw angle through spectral analysis. Masoud and Ali [18] harnessed 15 types of shelter designs, simulated the CFD code through Lattice Boltzmann Method, and they also verified the simulation results, concluding that porous shelters can reduce the crosswind forces and momentums of the vehicle.

To sum up, fruitful studies have been conducted in the hunting stability items on straight and curved tracks where the nonlinear and linear factors are taken into consideration. In addition, the stability of railway vehicles under crosswinds is carried out in terms of steady and unsteady types, the influences of curve radius, suspension parameters and other factors. However, there are few studies about the hunting stability and the derailment safety under different crosswind velocity with actual wheel profiles that are changing with the increase of operating mileage, which is worthy of deeper study.

In this paper, a 17-DOF mathematical model for a typical high-speed vehicle is set up on the basis of Ref [19], considering the vehicle running on straight and curved tracks with a steady crosswind blowing to inner/outer rail. At the same time, different wheel profiles with the increase of operating distance are measured and transformed to the correlated wheel/rail contact geometry, which are also put into the numerical calculation. Based on Newton-Raphson iteration method and Lyapunov's indirect theory, the equilibrium position of the vehicle system is precisely determined and the stability of the system is decided through the eigenvalues of Jacobian matrix. Consequently, the critical speeds of vehicle with different wheel wear stages under crosswinds blowing to inner/outer rail are calculated and analyzed. Furthermore, the influences of super-elevation and radius of the curved track are also considered in the numerical simulation. Additionally, the derailment safety of the vehicle system is discussed through corresponding indexes on straight and curved tracks.

\section{Mathematical models}

\subsection{Wheel/rail contact geometry and interactive forces}

Figure 1 illustrates the relationship of contact geometry parameters between wheel and rail, which is considered as nonlinear functions of the lateral displacement $\left(y_{w}\right)$ of the wheelset, with the outline of the radius of wheel profile $\left(R w_{1}, R w_{2}\right)$, wheel rolling radius $\left(r_{1}, r_{2}\right)$, contact angle $\left(\delta_{1}, \delta_{2}\right)$ and wheelset roll angle $\left(\varphi_{w}\right)$. With the change of the wheel/rail profile in the process of operation due to wears, the contact geometry relationship will also vary, which is difficult to represent with a formula. However, 




Fig. 1 Wheel/rail contact geometry parameters

when considering the rigid contact of the wheel and rail, the wheel-rail contact geometry can be uniquely determined. As a result, the nonlinear wheel/rail contact numerical tables are used to calculate the actual contact geometry parameters, where the intermediate value of the contact parameters can be derived from linear interpolation, with all of the contact geometry parameters used in this paper are measured through field tracking tests.

When the wheel rolls on the rail, there is a relative motion between the pairs of them, which causes creep force. For each individual wheel, it will bear longitudinal, lateral creep forces and spin creep moment as an interaction to the rail. With the nonlinear expressions of longitudinal, lateral and spin creepages for the left $(j=1)$ and right wheel $(j=2)$ written as Eq. (1).

$\left\{\begin{array}{l}\xi_{x j}=1-r_{j} / r_{0}+(-1)^{j} a \dot{\psi}_{w} / v-(-1)^{j} a / R \\ \xi_{y j}=\left(\dot{y}_{w}-v \dot{\psi}_{w}\right) \cos \left(\delta_{j}-(-1)^{j} \varphi_{w}\right) \\ \xi_{s j}=(-1)^{j} \sin \left(\left(\delta_{j}-(-1)^{j} \varphi_{w}\right) / r_{0}+\left(\dot{\psi}_{w} / v-1 / R\right)\right.\end{array}\right.$

where $R$ represents the radius of the curve, $v$ denotes the vehicle forward speed and $r_{0}$ is the wheel nominal rolling radius.

In order to calculate the corresponding creep loads, Kalker's linear creep theory is applied initially, where $F_{c x i} /$ $F_{c y i} / M_{c z i}$ mean the longitudinal creep force, lateral creep force and spin creep moment respectively. Figure $2 \mathrm{dem}$ onstrates the definition of the wheel/rail contact point axes $o_{j}^{\prime} x_{j}^{\prime} y_{j}^{\prime} z_{j}^{\prime}$. Below are the expressions on computing creep forces and creepages.

$\left\{\begin{array}{l}F_{c x j}=-f_{11 j} \xi_{x j} \\ F_{c y j}=-f_{22 j} \xi_{y j}-f_{23 j} \xi_{s j} \\ M_{c z j}=f_{23 j} \xi_{y j}-f_{33 j} \xi_{s j}\end{array}\right.$

where $f_{11 j}, f_{22 j}, f_{23 j}$ and $f_{33 j}$ are the creep coefficients determined by wheel/rail contact geometry parameters and materials of wheel and rail.



Fig. 2 Wheel/rail contact forces

To improve the accuracy on calculating creep forces, Shen [20] made the modification to Eq. (2) based on the saturation effect of creepages, which realized the nonlinear creep force model. Consequently, the lateral forces (in y), vertical forces (in z) as well as yaw moments (about z) acting on the wheelset by the rails in the wheelset axes oxyz (Fig. 2) can be derived with the creep forces, creep moments and wheel/rail normal contact forces through coordinate transformation between axes $o_{j}^{\prime} x_{j}^{\prime} y_{j}^{\prime} z_{j}^{\prime}$ and oxyz, as shown in Eqs. (3) and (4).

$F_{c j}=\sqrt{F_{c x j}^{2}+F_{c y j}^{2}}$

$F c j= \begin{cases}\mu \cdot N j\left[\left(\frac{F_{c j}}{\mu \cdot N j}\right)-\frac{1}{3}\left(\frac{F_{c j}}{\mu \cdot N j}\right)^{2}+\frac{1}{27}\left(\frac{F_{c j}}{\mu \cdot N j}\right)^{3}\right] & \left(\frac{F_{c j}}{3 \mu \cdot N j} \leq 1\right) \\ \mu \cdot N j\left(\frac{F_{c j}}{\mu \cdot N j}>1\right) & \left(\frac{F_{c j}}{3 \mu \cdot N j}>1\right)\end{cases}$

The saturation coefficient $\varepsilon$ is defined as Eq. (5), where $N_{j}$ means the contact normal forces, and $\mu$ is the friction factor of the wheel/rail. Thus, the final creep forces and moments is obtained through Eq. (6), which can be transformed to the lateral force and yawing moment acting on the wheelsets.

$\varepsilon=\frac{F_{c j}^{\prime}}{F_{c j}}$

$\left\{\begin{aligned} F_{c x j}^{\prime} & =F_{c x j} \cdot \varepsilon \\ F_{c y j}^{\prime} & =F_{c y j} \cdot \varepsilon \\ M_{c z j}^{\prime} & =F_{c z j} \cdot \varepsilon\end{aligned}\right.$

\subsection{Centrifugal forces on curved track}

When the vehicle runs on a curved track at a constant forward speed $v$, each body of the vehicle system also moves at speed $v$ along the track and there is a centrifugal force acting 
on each body. As the outer rail is super-elevated relative to the inner rail with super-elevation $h$ or anglea, the centrifugal force can be partly balanced by the gravitational force of the body. The forces acting on each body are shown as Fig. 3 . The unbalanced centrifugal force in the lateral direction (in y) can be obtained as

$F_{g i}=m_{i} \frac{v^{2}}{R} \cos \alpha-m_{i} g \sin \alpha$

where $m_{i}(i=1-7)$ indicates the mass of the each rigid body, $\mathrm{R}$ means the radius of the curved track, $h$ and $a$ is the super-elevation value and angle of the outer rail.

\subsection{Crosswind loads}

In general, the high-speed vehicle will bear five aerodynamic loads under crosswinds, which are the lateral and lift forces, as well as the roll, pitch and yaw moments (about $x, y$ and $z$ ). At the same time, the crosswinds are divided into steady and unsteady loads in terms of time history. However, it is always the lateral and lift forces, rolling moment that are of great influence on the stability for high-speed trains [21]. Thus, only three of the aerodynamic loads are considered in this paper in the form of steady crosswind.

Figure 4 presents the status of a vehicle that is running on track and bearing the loads from the crosswinds in its left side. The lateral force $F_{L a}$ in the lateral direction, the lift $F_{L}$ in the vertical direction and the roll moment $M_{R}$ can be expressed as Eq. (8), with the above loads acting on car body.

$$
\left\{\begin{array}{l}
F_{L a}=\frac{1}{2} \cdot C_{L a} \cdot \rho \cdot\left(u^{2}+v^{2}\right) \cdot S \\
F_{L}=\frac{1}{2} \cdot C_{L} \cdot \rho \cdot\left(u^{2}+v^{2}\right) \cdot S \\
M_{R}=\frac{1}{2} \cdot C_{R} \cdot \rho \cdot\left(u^{2}+v^{2}\right) \cdot S \cdot I
\end{array}\right.
$$

where $C_{L a} C_{L}$, and $C_{R}$ are the aerodynamic coefficient corresponding to aerodynamic loads respectively, which can

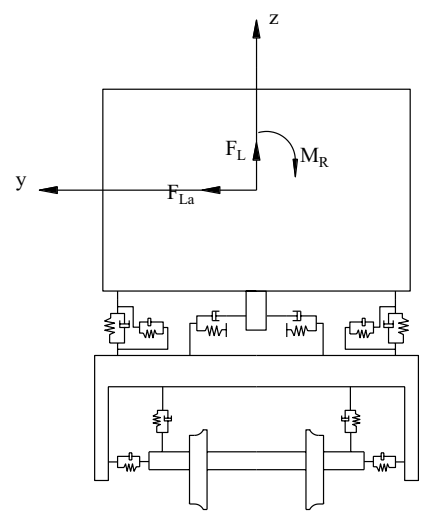

Fig. 4 Crosswind loads on car body

be obtained through the wind tunnel experiment [22] and fitted by polynomial as shown in Eq. (9), $\rho$ denotes the air density, $u$ and $v$ mean the crosswind velocity and vehicle speed respectively, $S$ is the frontal area and $/$ is frontal length.

$\left\{\begin{array}{l}C_{L a}=-0.9508 \cdot \psi^{3}+1.9252 \cdot \psi^{2}+0.3312 \cdot \psi+0.0276 \\ C_{L}=-0.9508 \cdot \psi^{3}+1.9252 \cdot \psi^{2}+0.3312 \cdot \psi-0.0624 \\ C_{R}=0.7222 \cdot \psi^{3}-1.3607 \cdot \psi^{2}-0.0393 \cdot \psi-0.0247\end{array}\right.$

where $\psi$ represents the sideslip angle between wind velocity and vehicle speed, as calculated in Eq. (10). Based on Eq. (10), the sideslip angles under specific crosswind velocity and vehicle speed are calculated as illustrated in Fig. 5. Apparently, the sideslip angle is positively correlated with wind velocity while negatively correlated with vehicle speed.

$\psi=\tan ^{-1} \frac{u}{v}$

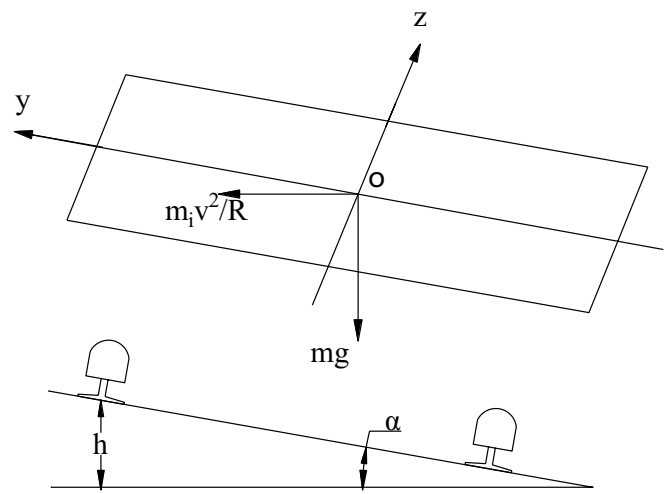

Fig. 3 Centrifugal forces on curved track

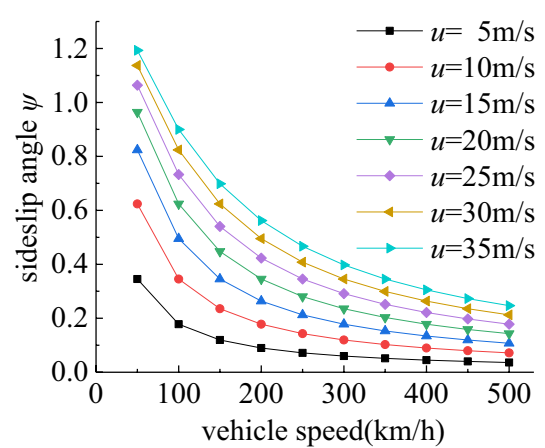

Fig. 5 Relationship between vehicle speed and sideslip angle 


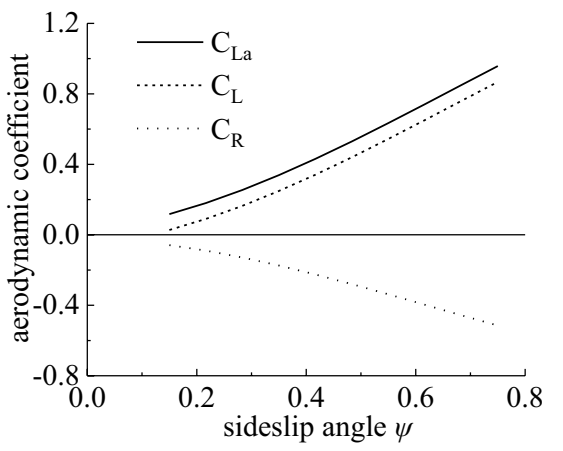

Fig. 6 Relationship between aerodynamic coefficients and sideslip angle

Table 1 Crosswind levels

\begin{tabular}{lll}
\hline Wind intensity & Wind scale & Wind velocity $(\mathrm{m} / \mathrm{s})$ \\
\hline 0 & Calm & $0-0.2$ \\
1 & Light air & $0.3-1.5$ \\
2 & Light breeze & $1.6-3.3$ \\
3 & Gentle breeze & $3.4-5.4$ \\
4 & Moderate breeze & $5.5-7.9$ \\
5 & Fresh breeze & $8.0-10.7$ \\
6 & Strong breeze & $10.8-13.8$ \\
7 & Moderate gale & $13.9-17.1$ \\
8 & Fresh gale & $17.2-20.7$ \\
9 & Strong gale & $20.8-24.4$ \\
10 & Whole gale & $24.5-28.4$ \\
11 & Storm & $28.5-32.6$ \\
12 & Hurricane & $32.6-36.9$ \\
$>12$ & Super typhoon & $>37.0$ \\
\hline
\end{tabular}

The relationship between the aerodynamic coefficients $C_{L a} C_{L}$, and $C_{R}$ and the sideslip angle are demonstrated in Fig. 6.

According to the corresponding standard [23], the crosswind is divided into different scale and intensity in terms of its velocity, which is illustrated in Table 1. In this paper, the crosswind velocity increases linearly from $0 \mathrm{~m} / \mathrm{s}$ to $35 \mathrm{~m} / \mathrm{s}$, since the intensity exceeded 12 hardly happens, it is not considered during the calculation.

\subsection{Motion differential equation of the vehicle system}

A nonlinear mathematical model is utilized to analyze the stability of a typical high-speed vehicle, which is shown in Fig. 7. The vehicle system is taken as a multi-rigid-body system composed of car body, bogie frames and wheelsets, where the car body/bogie frames are linked by the secondary suspensions and the bogie frames/wheelsets are connected via the primary suspension system. Besides, the suspension system is viewed as linear while the wheel/ rail interactive forces are nonlinear.

The coordinate system of the vehicle model is established in Fig. 7, where the $x$ direction is for the forward speed, $y$ axis is parallel to the track plane and directed to the left of the track and the $z$ axis is normal to the plane of the track and straight up. In view of the weak-coupling relationship between the vertical and lateral vibrations of the vehicle system, only the lateral vibration is considered to analyze the stability. The total number of degrees of freedom of the vehicle system is 17 , which is listed in Table 2.

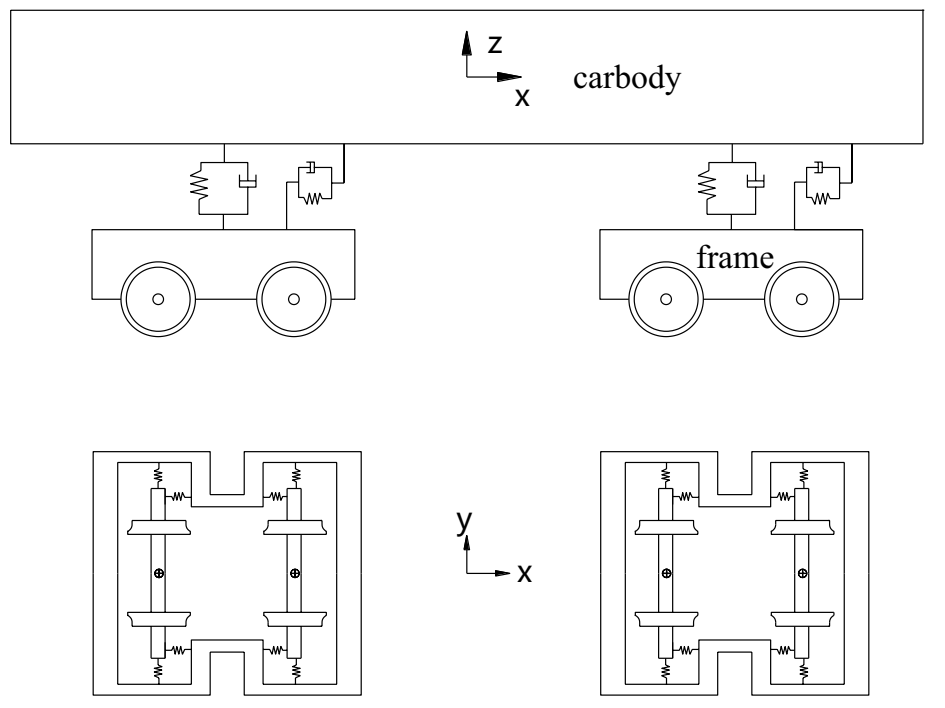

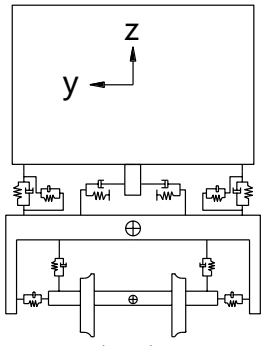

wheelset

Fig. 7 High-speed vehicle model 
Table 2 Degrees of freedom of the vehicle system

\begin{tabular}{llll}
\hline Components & Lateral & Yaw & Roll \\
\hline Car body & $y_{c}$ & $\psi_{c}$ & $\theta_{c}$ \\
Bogie frames $(\mathrm{i}=1,2)$ & $y_{b i}$ & $\psi_{b i}$ & $\theta_{b i}$ \\
Wheelsets $(\mathrm{j}=1-4)$ & $y_{w j}$ & $\psi_{w j}$ & \\
\hline
\end{tabular}

Then, the motion differential equation of the vehicle system on an ideal smooth curved track can be derived and expressed as the following vector form.

$M \ddot{y}+C \dot{y}+K y=F_{c}(\dot{y}, y, v, R, h)+F_{g}(v, R, h)+F_{w}(v, u)$

where $y$ is coordinate vector and $M, C, K$ are diagonal mass matrix, damping matrix and stiffness matrix respectively. $F_{c}$ denotes the nonlinear force vector consisting of the wheel/rail lateral force and yaw moment acting on wheelsets, $F_{g}$ represents the unbalanced centrifugal force on each body and $F_{w}$ means the crosswind loads on car body.

Through coordinate transformation $x=(\dot{y}, y)^{T}$, the second order differential Eq. (11) can be transformed into the following equation

$\frac{d x}{d t}=f(x, v, R, h)$

and the linearized equation is as below

$\frac{d x}{d t}=A x$

where $A=\left[\frac{\partial f}{\partial x}\right]_{x=x^{*}}$ is the Jacobian matrix at the equilibrium position $x^{*}=\left(0, y^{*}\right)^{T}$.

\section{Determination of critical speed}

To acquire the critical speed of the vehicle, the equilibrium position of the system must be attained at first. As we know, the equilibrium position of the vehicle running on straight track is equal to $x=0$, while it is unknown on curved track. Hence, the equilibrium point should be obtained primarily and then the eigenvalues of the system can be calculated. When considering the vehicle running on a straight track under crosswinds, it just needs to set the curve radius $\mathrm{R} \rightarrow \infty$, and the super-elevation $\mathrm{h}=0$.

To calculate the equilibrium position of the vehicle system, we can set the velocity and acceleration vectors $\dot{y}=\ddot{y}=0$ in Eq. (11), and then obtain the following nonlinear algebraic equation

$H(y)=K y-F_{c}(y, v, R, h)-F_{g}(v, R, h)-F_{w}(v, u)=0$
The Newton-Raphson iteration method is used to solve the above nonlinear algebraic equations, with the acquisitions of the equilibrium position of the vehicle system $\left(y=y^{*}\right)$.

Then, On the basis of the Lyapunov's indirect method, the stability of a nonlinear system near its equilibrium point can be determined by the eigenvalues of Jacobian matrix $A$. If all the real parts of the eigenvalues are negative, then the system is asymptotically stable, while it is unstable when there is at least one eigenvalue with positive real part. Hence, the critical speed of the vehicle system is defined as the speed at which a pair of complex conjugate eigenvalues have zero real parts while the other eigenvalues have negative real parts.

Based on the above-mentioned theories, a computational program is written to calculate the critical speed with corresponding parameters. To verify the program, the comparison was conducted with Ref $[2,14]$ using the same parameters, where the load condition was set as without crosswind as same as Ref [2]. Thus, the critical speed of the vehicle system can be calculated. Figure 8 shows an example for the determination of the critical speed. It can be seen that with the increase of vehicle speed, a pair of complex conjugate eigenvalues cross the imaginary axis at the critical speed $334 \mathrm{~km} / \mathrm{h}$.

Through the simulation, the error percentage between the Ref [2] and this paper can be attained as shown in Table 3.

From Table 3, we can see that the error percentage is $1.37 \%$, which proves the program to be right. Then, the simulation can be conducted to analyze the critical speed of vehicle under different conditions.



Fig. 8 Determination of critical speed 
Table 3 Comparison of critical speeds

\begin{tabular}{ll}
\hline & Speed $(\mathrm{km} / \mathrm{h})$ \\
\hline Ref [12] & 329.5 \\
This paper & 334 \\
Error percentage & $1.37 \%$ \\
\hline
\end{tabular}

\section{Numerical simulation and analysis}

\subsection{Hunting stability under crosswinds}

\subsubsection{Influences of wheel profile wears}

Given that the wheel profile will change due to wear with the increase of the operating mileage, and alter the relationship of wheel/rail contact geometry parameters, a typical high-speed vehicle has been utilized to monitor the wheel profiles under each operating stage. Figure 9 illustrate the measured wheel profiles at three stage, i.e., the new, mid-worn and end-worn periods at 150,000 km and 250,000 operating mileages respectively. Thus, the equivalent conicity can be calculated on account of each individual wheel profile according to UIC519 [24], as shown in Fig. 10. It can be seen from Figs. 9 and 10 that both the wheel wear and equivalent conicity are augmented with the incremental operating mileage.

Assuming that the vehicle is running on straight track, the critical speeds at different crosswind velocity $(0-35 \mathrm{~m} / \mathrm{s})$ under three stages of wheel profiles are calculated and shown as Fig. 11. At the same time, the corresponding lateral displacement of the leading wheelset and equivalent conicity are illustrated in Fig. 12. There is no doubt that the critical speed in the three periods is decreasing with the increase of the crosswind velocity, from $418 \mathrm{~km} / \mathrm{h}$ without crosswind till about $310 \mathrm{~km} / \mathrm{h}$ at crosswind velocity $35 \mathrm{~m} / \mathrm{s}$, which has a prominent decline

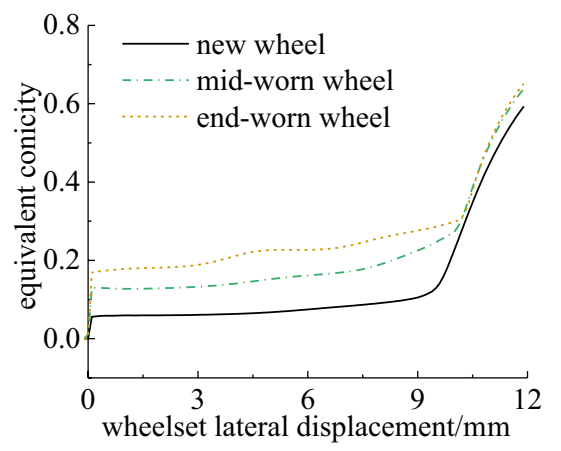

Fig. 10 Equivalent conicity

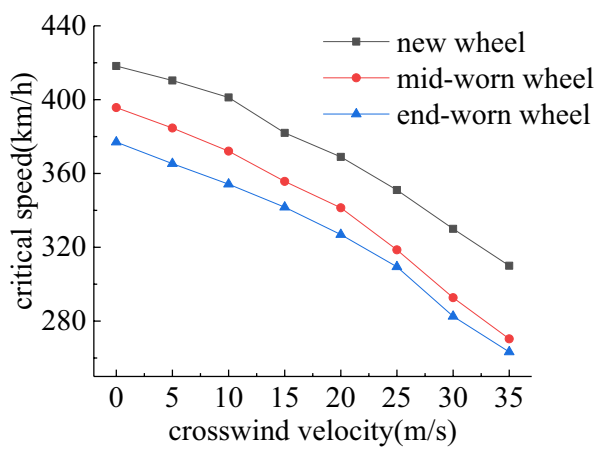

Fig. 11 Influence of wheel wear on critical speed

by $25.8 \%$ in new wheel condition. In addition, the critical speed in the new wheel status is higher than that of other two worn-wheel conditions, which is resulted from the increase of the equivalent conicity in the worn stages as shown in Fig. 11. Furthermore, the gap between nonwind $(u=0)$ and that under $u=25 \mathrm{~m} / \mathrm{s}$ or even higher are apparently widen during two worn periods, indicating that crosswind loads especially for strong winds does have a prominent effect on the stability of the vehicle after the
Fig. 9 Measured wheel profiles

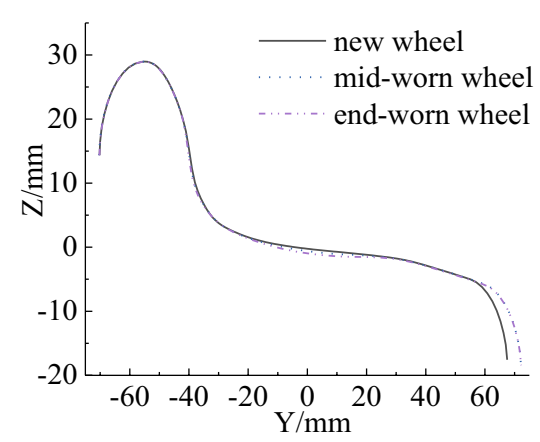

(a) Wheel profile

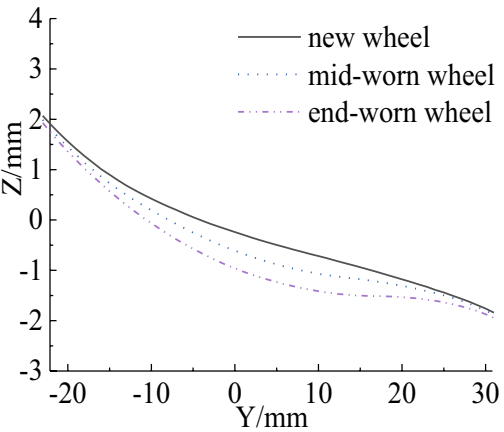

(b) Local enlargement

SN Applied Sciences A SPRINGer NATURE journa 
occurrence of wheel wears. Lastly, under the identical wheel wear state, the change of crosswind velocity has few effects on the equivalent conicity.

\subsubsection{Influences of wind direction on curved track}

Figure 4 demonstrates the crosswind forces on the vehicle blowing from inner to outer rail, while the lateral force and the roll moment should be reverse as the crosswind
Fig. 12 Lateral displacement and equivalent conicity under crosswinds on straight track

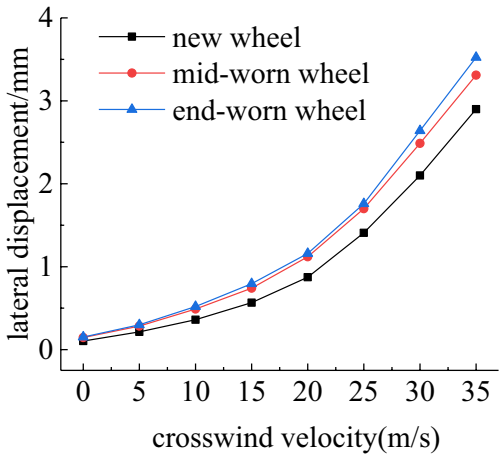

(a) Lateral displacement



(b) Equivalent conicity
Fig. 13 Critical speed under crosswinds blowing to a inner rail, $\mathbf{b}$ outer rail



(a)

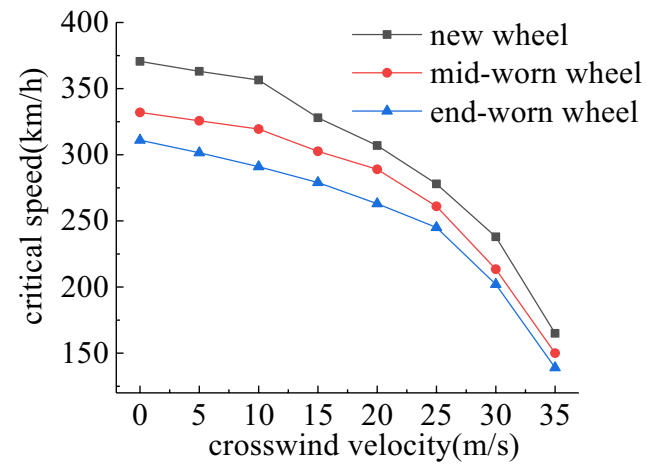

(b)

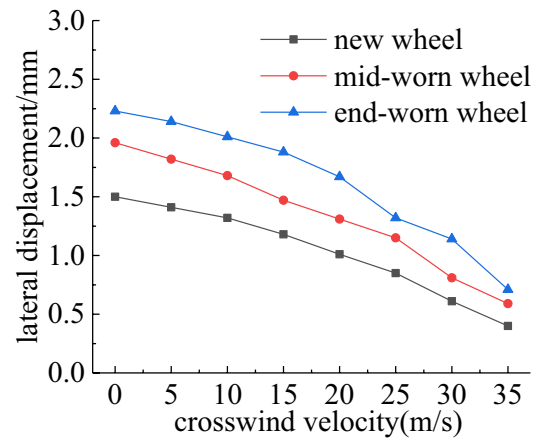

(a) Lateral displacement

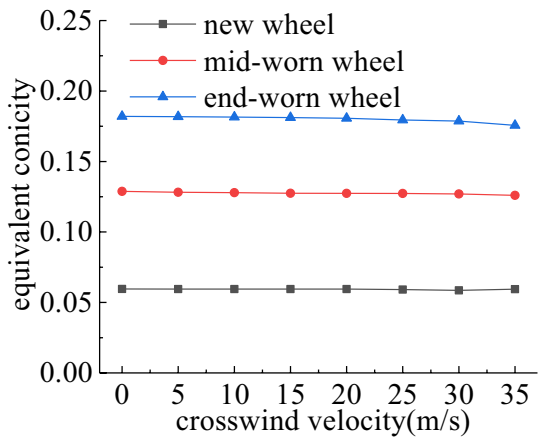

(b) Equivalent conicity

Fig. 14 Lateral displacement and equivalent conicity under crosswinds blowing to inner rail 
Fig. 15 Lateral displacement and equivalent conicity under crosswinds blowing to outer rail



(a) Lateral displacement

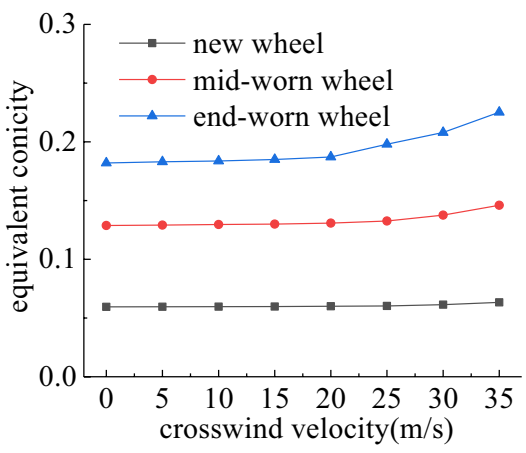

(b) Equivalent conicity is blowing from outer to inner rail, which can greatly influence the equilibrium position of vehicle. To illustrate the relationship between the critical speed of the vehicle and crosswind velocity on the basis of the wind direction, the conditions under crosswind blowing to outer/inner rail in different wheel worn periods are shown in Fig. 13a, b respectively, with curve radius $R=5000 \mathrm{~m}$, super-elevation $\mathrm{h}=120 \mathrm{~mm}$, followed by calculating results of wheelset lateral displacement and equivalent conicity in Figs. 14 and 15 .

It can be seen that when the crosswind blows to the inner rail, the critical speed increases while the lateral displacement reduces as the crosswind velocity is increasing. However, the tendency of that is opposite when the crosswind blows to the outer rail. Thus, the case that crosswind blows to outer rail should be considered seriously, which is the most dangerous load condition. What's more, the case that crosswind is blowing to outer track has predominant effects on the critical speed, because the change of the critical speed is much more obvious under the same wind velocity augmentation.

\subsubsection{Influences of curve radius and super-elevation}

The influences of curve radius $\mathrm{R}$ ranging from 5000 to $8000 \mathrm{~m}$ with $\mathrm{h}=120 \mathrm{~mm}$ and super-elevation $\mathrm{h}$ from 100 to $160 \mathrm{~mm}$ with $\mathrm{R}=5000 \mathrm{~m}$ on the vehicle critical speed during the mid-worn period considering crosswinds blowing to the outer track are revealed in Fig. 16a, b. It is clear from the figures that both the curve radius and superelevation have an eminent impact on the critical speed of the vehicle system, which is caused by the variation of the creep forces in wheel/rail interaction as well as the aerodynamic loads. That is to say, as the train is running under a specific crosswind velocity in a certain worn-wheel period, the larger the curve radius and super-elevation are, the higher the critical speed will be, bringing a smaller wheelset lateral displacement as shown in Figs. 17 and 18. Additionally, the equivalent conicity remains steady regardless of crosswind velocity during the same worn wheel period.

\subsection{Derailment safety under crosswinds}

The derailment safety of high-speed trains running on straight and curved tracks (with curve radius $R=5000 \mathrm{~m}$,
Fig. 16 Influence of a R on critical speed, $\mathbf{b}$ h on critical speed

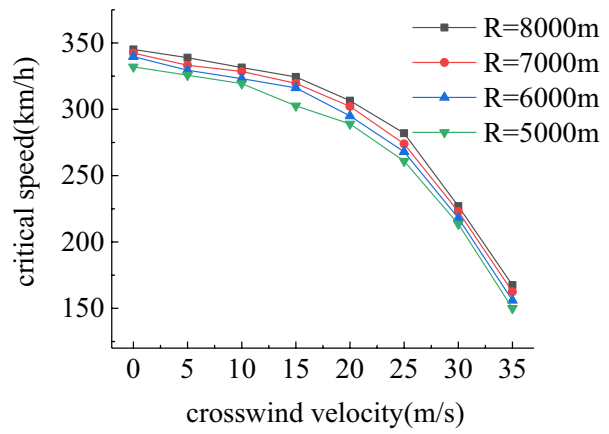

(a)

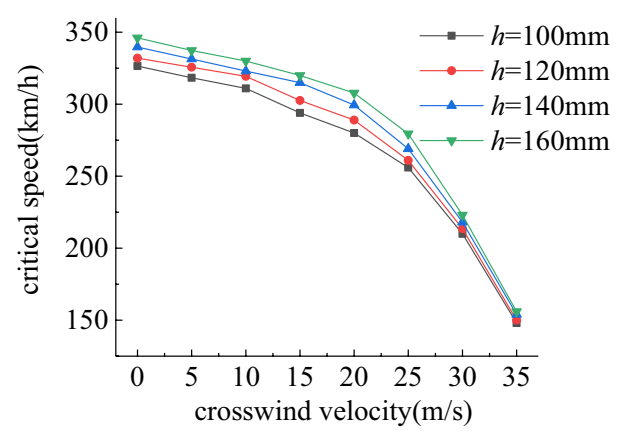

(b) 
Fig. 17 Lateral displacement and equivalent conicity under crosswinds considering $R$

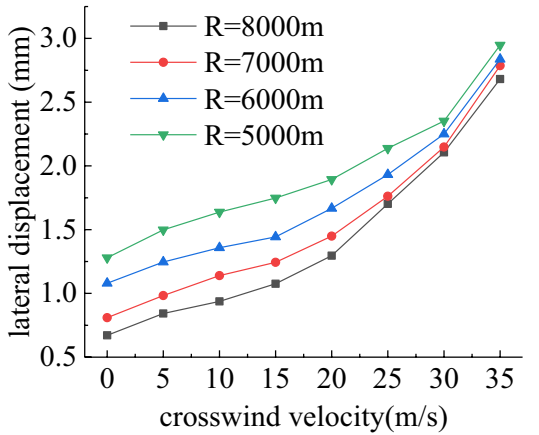

(a) Lateral displacement

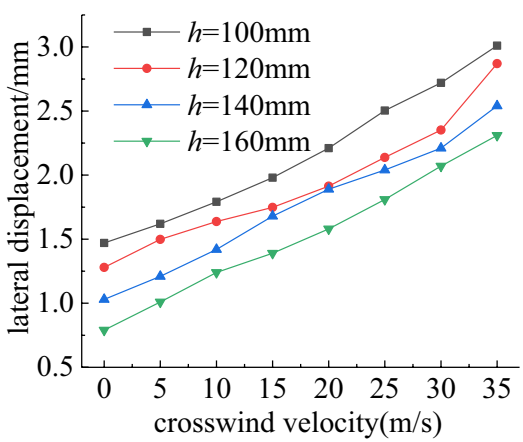

(a) Lateral displacement

Fig. 19 Derailment criterions for vehicle running on straight track

Fig. 18 Lateral displacement and equivalent conicity under crosswinds considering $\mathrm{h}$



(a) Derailment coefficient

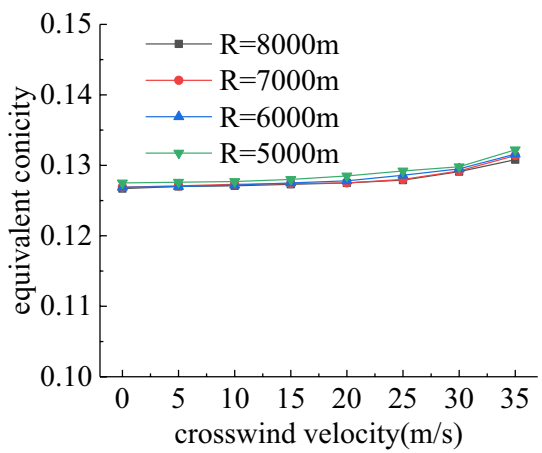

(b) Equivalent conicity



(b) Equivalent conicity

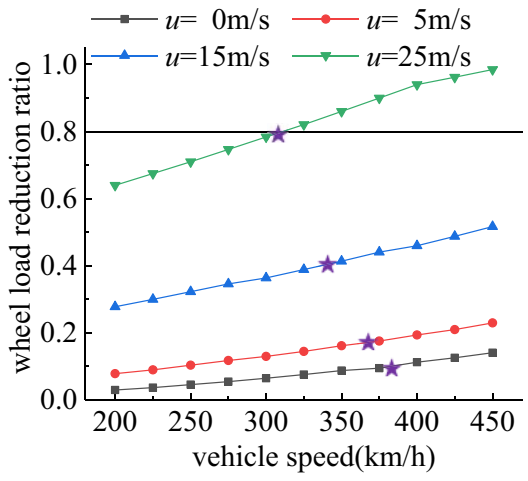

(b) Wheel load reduction ratio

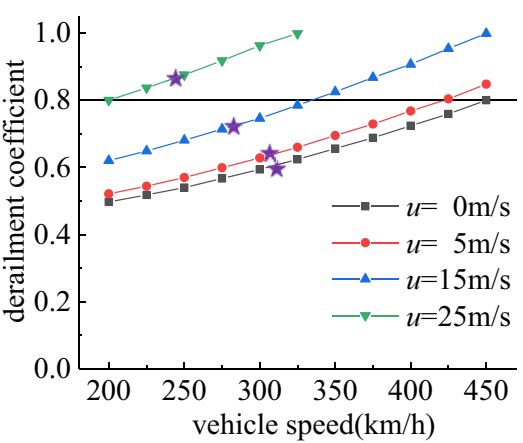

(a) Derailment coefficient

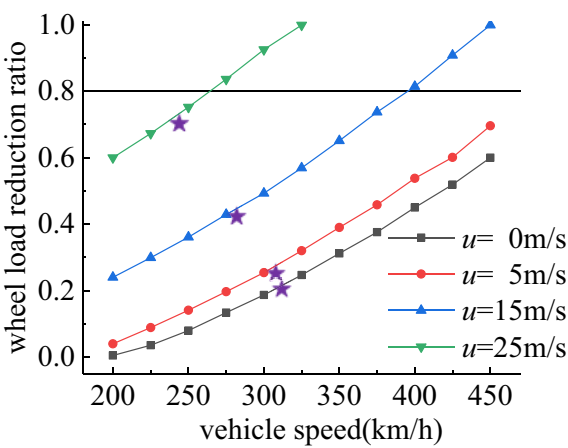

(b) Wheel load reduction ratio 
super-elevation $\mathrm{h}=120 \mathrm{~mm}$ ) under crosswinds blowing from inner to outer rail, which is the most dangerous case, is also studied. As the derailment coefficient (defined as wheel lateral force over vertical force) and wheel load reduction ratio (defined as vertical load difference between left and right wheels over average vertical load) are the essential criterions for evaluating the running safety of railway vehicles, Figs. 19 and 20 illustrate the two indexes for the high-speed vehicle in the end-worn period running between 200 and $450 \mathrm{~km} / \mathrm{h}$, where the corresponding critical speeds of the vehicle are listed in pentagrams. Evidently, the values of derailment coefficient and wheel load reduction ratio increase with the incremental vehicle speed in spite of the running tracks, however, when running on curved track considering crosswind blowing to outer rail, the growth trend is more obvious than that of straight line. Furthermore, the values of derailment coefficient and wheel load reduction ratio are lower in the straight track during the same vehicle speed, which indicates that the aerodynamic loads on curved track should be treated well since it can greatly influence the derailment safety of railway vehicles. In addition, when the vehicle is running under the condition of lower crosswind velocity $(u \leq 15 \mathrm{~m} / \mathrm{s})$, the derailment safety indexes always meet the requirements no matter what the track is, while it should be limited in the strong crosswinds as the values are out of limitation (0.8) under the corresponding critical speed $(v=309.4 \mathrm{~km} / \mathrm{h}$ on straight line, $v=245 \mathrm{~km} / \mathrm{h}$ on curved lines). Finally, when assessing the derailment safety for vehicle systems, it is the derailment coefficient that is more reliable than wheel load reduction ratio, which is stricter in terms of the standard limitation.

\section{Conclusions}

The hunting stability and derailment safety of high-speed trains on straight and curved tracks under steady -crosswinds has been studied through numerical simulation. The 17-DOF vehicle system model hypothesizes the factors of wheel/rail forces, crosswind loads and centrifugal forces as nonlinear, while the suspension system is considered as linear. During the simulation, the wheel profiles are measured and transformed to the correlated wheel/rail contact geometry. Also, eight kinds of crosswind velocity are considered in the paper. The work of the paper can be concluded as follows:
1. The wheel profile alters with the increase of operating mileage, which changes the wheel/rail contact geometry, leading to larger equivalent conicity. During the identical worn wheel condition, the change of crosswind velocity has tiny influences on the equivalent conicity.

2. It is the end-worn wheel period that has a significant influence on the critical speed. The increase of crosswind velocity becomes a dominant factor in reducing the stability of vehicle system.

3. When the crosswind blows to the inner rail, the critical speed increases as the crosswind velocity is increasing while it decreases as the crosswind blows to outer rail. Thus, the latter load condition should be taken seriously when considering the hunting stability of railway vehicles.

4. Both the curve radius and super-elevation can greatly affect the critical speed. The higher curve radius and super-elevation the vehicle undergoes, the more stable the system will be.

5. When considering the derailment safety of high-speed trains, the crosswind on curved track should be treated seriously as it can greatly affect the derailment criterions, especially when the crosswind velocity exceeds $15 \mathrm{~m} / \mathrm{s}$. In addition, the derailment coefficient is more precise than wheel load reduction ratio, which should be taken as the limitation.

In the further research, the influences of unsteady crosswinds should be considered and the complex conjugate eigenvalues can be analyzed from the frequency domain.

Funding This work was supported by the projects of National Natural Science Foundation of China (Grant No. 11790282), the Program of State Key Laboratory of Traction Power (Grant No. 2019TPL_T20) and the Fundamental Research Funds for the Central Universities (Grant No. 2682017CX010).

\section{Compliance with ethical standards}

Conflict of interest We declare that we have no conflict of interest to this work.

\section{Appendix}

See Table 4. 
Table 4 Parameters of vehicle

\begin{tabular}{|c|c|}
\hline Parameters & Value \\
\hline Mass of car body & $M_{c}=34,689 \mathrm{~kg}$ \\
\hline Mass of bogie frame & $M_{b}=2758 \mathrm{~kg}$ \\
\hline Mass of wheelset & $M_{w}=1524 \mathrm{~kg}$ \\
\hline Roll inertia of car body & $I_{c x}=61,000 \mathrm{~kg} / \mathrm{m}^{-2}$ \\
\hline Yaw inertia of car body & $I_{c z}=1,816,000 \mathrm{~kg} / \mathrm{m}^{-2}$ \\
\hline Roll inertia of bogie frame & $I_{b x}=1760 \mathrm{~kg} / \mathrm{m}^{-2}$ \\
\hline Yaw inertia of bogie frame & $I_{b z}=4000 \mathrm{~kg} / \mathrm{m}^{-2}$ \\
\hline Yaw inertia of wheelset & $I_{w z}=910 \mathrm{~kg} / \mathrm{m}^{-2}$ \\
\hline Primary longitudinal stiffness & $K_{p x}=8633 \mathrm{kN} / \mathrm{m}$ \\
\hline Primary lateral stiffness & $K_{p y}=4864 \mathrm{kN} / \mathrm{m}$ \\
\hline Primary vertical stiffness & $K_{p z}=900 \mathrm{kN} / \mathrm{m}$ \\
\hline Secondary longitudinal stiffness & $K_{s x}=162 \mathrm{kN} / \mathrm{m}$ \\
\hline Secondary lateral stiffness & $K_{s y}=162 \mathrm{kN} / \mathrm{m}$ \\
\hline Secondary vertical stiffness & $K_{s z}=227 \mathrm{kN} / \mathrm{m}$ \\
\hline Primary vertical damping & $C_{p z}=17.7 \mathrm{kNs} / \mathrm{m}$ \\
\hline Secondary longitudinal damping & $C_{s x}=360 \mathrm{kNs} / \mathrm{m}$ \\
\hline Secondary lateral damping & $C_{s y}=22 \mathrm{kNs} / \mathrm{m}$ \\
\hline Secondary vertical damping & $C_{s z}=20 \mathrm{kNs} / \mathrm{m}$ \\
\hline Center distance of car body coupler & $L_{c}=25 \mathrm{~m}$ \\
\hline Center distance of bogie & $L_{s}=18 \mathrm{~m}$ \\
\hline Distance of car body C.G. to secondary suspension & $H_{1}=1.107 \mathrm{~m}$ \\
\hline Distance of frame C.G. to secondary suspension & $H_{2}=0.18 \mathrm{~m}$ \\
\hline Distance of wheelset C.G. to primary suspension & $H_{3}=0.15 \mathrm{~m}$ \\
\hline Wheel rolling radius & $R=0.445 \mathrm{~m}$ \\
\hline Half lateral distance of wheel/rail contact points & $a=0.7465 \mathrm{~m}$ \\
\hline Density of air & $\rho=1.205 \mathrm{~kg} / \mathrm{m}^{3}$ \\
\hline Frontal area & $A=25 \mathrm{~m}^{2}$ \\
\hline Frontal length & $I=1.992 \mathrm{~m}$ \\
\hline
\end{tabular}

\section{References}

1. Zeng J, Pingbo W (2004) Stability analysis of high speed railway vehicles. JSME Int J Ser C 47:464-470

2. Zeng X, Han W, Lai J, Sheng H (2016) Hunting stability of highspeed railway vehicles on a curved track considering the effects of steady aerodynamic loads. J Vib Control 22(20):4159-4175

3. Baker C, Cheli F, Orellano A, Paradot N, Proppe C, Rocchi D (2009) Cross-wind effects on road and rail vehicles. Veh Syst Dyn 47(8):983-1022

4. Kim P, Jung J, Seok J (2011) A parametric dynamic study on hunting stability of full dual-bogie railway vehicle. Int J Precisi Eng Manuf 12:505-519

5. Cheng YC, Lee SY (2010) Stability analysis of high-speed railway vehicle using half-car model. Int J Heavy Veh Syst 17:139-158

6. Zboinski K, Dusza M (2011) Extended study of railway vehicle lateral stability in a curved track. Veh Syst Dyn 49:789-810

7. Deng E, Yang W, Lei M et al (2019) Aerodynamic loads and traffic safety of high-speed trains when passing through two windproof facilities under crosswind: a comparative study. Eng Struct 188:320-339
8. Cheng YC, Huang $\mathrm{CH}$, Kuo CM, Cheng $\mathrm{CH}$ (2013) Derailment risk analysis of a tilting railway vehicle moving over irregular tracks under wind loads. Int J Struct Stab Dyn 13(8):1350038

9. Baker C, Hemida H, Iwnicki S et al (2011) Integration of crosswind forces into train dynamic modelling. Proc Inst Mech Eng Part F J Rail Rapid Transit 225:154-164

10. Andreas H, Bernhard K, Tilman B et al (2014) Considerations on active control of crosswind stability of railway vehicles. Veh Syst Dyn 52(6):759-775

11. Kim MS, Kim GY, Kim HT et al (2016) Theoretical cross-wind speed against rail vehicle derailment considering the crossrunning wind of trains and the dynamic wheel-rail effects. J Mech Sci Technol 30(8):3487-3498

12. Choi* Y-S, Shin B-S (2015) Critical speed of high-speed trains considering wheel-rail contact. J Mech Sci Technol 29(11):4593-4600

13. Yan N, Chen X, Li Y (2018) Assessment of overturning risk of high speed trains in strong crosswinds using spectral analysis approach. J Wind Eng Ind Aerodyn 174:103-118

14. Zeng $X-H$, Lai J, Han W (2018) Hunting stability of high-speed railway vehicles under steady aerodynamic loads. Int J Struct Stab Dyn 18(11):1850093 
15. Mao J, Xi YH, Yang GW (2011) Research on influence of characteristics of cross wind field on aerodynamic performance of a high-speed train. J China Railw Soc 4:22-30 (in Chinese)

16. Kikuchi K, Suzuki M (2015) Study of aerodynamic coefficients used to estimate critical wind speed for vehicle overturning. J Wind Eng Ind Aerodyn 147:1-17

17. Zhuang Y, Xiyun L (2015) Numerical investigation on the aerodynamics of a simplified high-speed train under crosswinds. Theor Appl Mech Lett 5:181-186

18. Masoud M, Ali RM (2019) Analysis of the effects of lateral wind on a high speed train on a double routed railway track with porous shelters. J Wind Eng Ind Aerodyn 184:116-127

19. Zeng J (2001) Numerical analysis of nonlinear stability for railway passenger cars. Chin J Mech Eng (English Ed) 14(2):97-101

20. Shen ZY, Hedrick JK, Elkins JA (1983) A comparison of alternative creep force models for rail vehicle dynamic analysis. Veh Syst Dyn 12:79-83
21. Guang-jun GAO, Hong-qi TIAN, Song YAO, Tang-hong LIU, Guang-hong BI (2004) Effect of strong cross-wind on the stability of trains running on the Lanzhou-Xinjiang Railway Line. J China Railw Soc 4:36-40 (In Chinese)

22. Train operating safety under transient wind load: Miao Xiujuan: Central South University (2011)

23. GB/T 28591-2012 (2012) Wind scale. China National Standardization Administration (in Chinese)

24. UIC519-2004 (2004) Method for determining the equivalent conicity. International Union of Railways

Publisher's Note Springer Nature remains neutral with regard to jurisdictional claims in published maps and institutional affiliations. 